\title{
Precision tests of nonadiabatic perturbation theory with measurements on the DT molecule
}

\author{
K.-F. Lai, ${ }^{1}$ P. Czachorowski, ${ }^{2}$ M. Schlösser, ${ }^{3}$ M. Puchalski, ${ }^{4}$ J. Komasa, ${ }^{4}$ K. Pachucki, ${ }^{2}$ W. Ubachs, ${ }^{1}$ and E. J. Salumbides $\odot^{1,}{ }^{*}$ \\ ${ }^{1}$ Department of Physics and Astronomy, LaserLaB, Vrije Universiteit Amsterdam, De Boelelaan 1081, 1081 HV Amsterdam, Netherlands \\ ${ }^{2}$ Faculty of Physics, University of Warsaw, Pasteura 5, 02-093 Warsaw, Poland \\ ${ }^{3}$ Tritium Laboratory Karlsruhe, Institute of Technical Physics, Karlsruhe Institute of Technology, Hermann-von-Helmholtz-Platz, 1 , \\ 76344 Eggenstein-Leopoldshafen, Germany \\ ${ }^{4}$ Faculty of Chemistry, Adam Mickiewicz University, Umultowska 89b, 61-614 Poznań, Poland
}

(Received 29 March 2019; revised manuscript received 24 September 2019; published 22 November 2019)

\begin{abstract}
First-principles calculations are presented for fundamental vibrational splitting energies of tritium-bearing molecular hydrogen species with the improved treatment of the nonrelativistic, relativistic, and quantum electrodynamic energy contributions resulting in a total uncertainty of $0.00011 \mathrm{~cm}^{-1}$ for DT, or about a 100 times improvement over previous results. Precision coherent Raman spectroscopic measurements of $Q(J=0-5)$ transitions in DT were performed at an accuracy of $<0.0004 \mathrm{~cm}^{-1}$, representing an even larger 250-fold improvement over previous experiments. Perfect agreement between experiment and theory is found, within $1 \sigma$, for all six transitions studied.
\end{abstract}

DOI: 10.1103/PhysRevResearch.1.033124

\section{INTRODUCTION}

Seventy years after its development, quantum electrodynamics (QED) has emerged to be the best-tested theory in physics. While QED is most accurately tested from the measurement of the anomalous magnetic moment of a free electron [1] and atomic recoil measurements [2,3], tests in the bound atomic hydrogen system are at present limited by effects of the proton structure [4,5]. Precision tests in the hydrogen atom rely ultimately on the narrowest transition involving the long-lived $2 S$ quantum state with a natural lifetime of $0.12 \mathrm{~s}$ [6]. In contrast, the additional rotational and vibrational degrees of freedom in $\mathrm{H}_{2}$ give rise to a multitude of states in the ground electronic manifold with extremely long lifetimes on the order of $10^{5}-10^{6} \mathrm{~s}$ [7]. In addition, access to all six isotopic variants of molecular hydrogen enables a robust validation of nonadiabatic perturbation theory.

The additional complexity of the four-body molecular hydrogen system presents formidable challenges in firstprinciples calculations. The last decade has shown great improvements in calculation of the nonrelativistic energies using a perturbative approach $[8,9]$, or a recent nonperturbative treatment [10]. Concurrent developments in the calculation of relativistic [11], QED [12], and associated recoil corrections have led to sub-MHz accuracies in level energies of the stable molecular hydrogen species $\mathrm{H}_{2}, \mathrm{HD}$, and $\mathrm{D}_{2}$ [13], but have not been applied to tritiated isotopologues until now.

\footnotetext{
*e.j.salumbides@vu.nl

Published by the American Physical Society under the terms of the Creative Commons Attribution 4.0 International license. Further distribution of this work must maintain attribution to the author(s) and the published article's title, journal citation, and DOI.
}

Parallel progress in experiments has resulted in an accurate measurement of the dissociation energy of the $\mathrm{H}_{2}$ molecule $[14,15]$, the measurement of its fundamental vibrational splitting [16], as well as very weak quadrupole overtone transitions [17-19]. Similar spectroscopic studies have also been applied to the other stable $\mathrm{D}_{2}$ (cf. [20-22]) and the mixed isotopologue HD (cf. [23-26]). On the other hand, very few precision studies have been undertaken on the tritium-bearing species [27-29], on account of difficulties in handling radioactive tritium. If these practical challenges were overcome, access to tritium-bearing species $\mathrm{T}_{2}, \mathrm{HT}$, and DT would double the number of molecular hydrogen test systems.

Here we present highly accurate calculations of the rovibrational transitions for all tritiated molecular hydrogen that are two orders of magnitude more accurate than previous studies [30]. This improvement is obtained by applying a recently developed nonadiabatic perturbation theory approach to obtain accurate nonrelativistic energies [9], as well as by systematic treatment of leading-order, higher-order, and recoil relativistic and QED corrections based on nonrelativistic wave functions. The calculations are benchmarked by accurate measurements of DT transition energies, which enables precision tests in tritiated species that are now sensitive to QED effects.

\section{EXPERIMENT}

As for the experimental study, we use the nonlinear frequency-mixing scheme of coherent anti-Stokes Raman spectroscopy (CARS), illustrated in the inset of Fig. 1. An anti-Stokes coherent beam is produced for signal detection, at frequency $\omega_{\mathrm{AS}}=2 \omega_{P}-\omega_{S}$ corresponding to $\lambda_{\mathrm{AS}} \sim 464 \mathrm{~nm}$, whenever the frequency difference between the pump $\left(\omega_{P}\right)$ and Stokes $\left(\omega_{S}\right)$ frequencies is in resonance with a vibrational mode $\left(\omega_{R}\right)$ in the molecule. The high-resolution CARS setup has been described in a previous work on $\mathrm{T}_{2}$ [31]. An injection-seeded and frequency-doubled Nd:YAG laser 


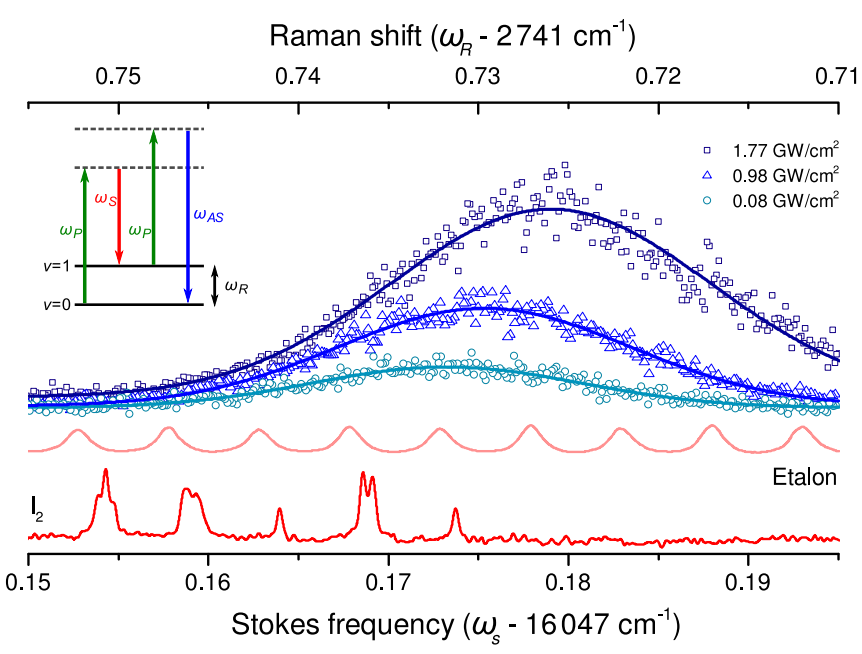

FIG. 1. $Q(1)$ transition of the DT $X^{1} \Sigma_{g}^{+}(v=0 \rightarrow 1)$ Raman band, probed at different peak intensities and plotted with respect to the Stokes frequency $\omega_{S}$ (lower frequency axis) and the Raman shift $\omega_{R}$ (upper axis). The solid lines through the DT data points are Gaussian fits, with the line centers exhibiting ac-Stark shifts. The transmission peaks of the stabilized etalon and saturated $I_{2}$ spectrum used in the relative and absolute frequency calibrations of $\omega_{S}$ are also plotted. Inset: Nonlinear four-wave mixing scheme.

provides the pump beam $\left(\lambda_{P} \sim 532 \mathrm{~nm}\right)$, while the Stokes radiation $\left(\lambda_{S} \sim 623 \mathrm{~nm}\right)$ originates from a narrow-band pulsed dye amplifier (PDA) system [32], which is seeded by a continuous-wave $(\mathrm{cw})$ ring dye laser. DT at a partial pressure of 4 mbar is contained in a $4-\mathrm{cm}^{3}$ gas cell, prepared from a 4:1 mixture of $\mathrm{D}_{2}$ and $\mathrm{T}_{2}$ at the Tritium Laboratory Karlsruhe and transported to LaserLaB Amsterdam for the spectroscopic measurements [33].

Recordings of the $Q(1)$ Raman transition at different intensities are shown in Fig. 1, manifesting ac-Stark broadening and shifting. The cw-seed frequency for the $\omega_{S}$ radiation is calibrated using a HeNe-stabilized etalon in combination with an absolute frequency reference from saturation $I_{2}$ spectroscopy [34]. The cw-pulse frequency offset induced by frequency chirp effects in the pulsed-dye amplification $[32,35,36]$ is measured and corrected for [37]. The frequency of the $\omega_{P}$ pulse is monitored online using a highresolution wavemeter (High Finesse Ångstrom WSU-30), which is periodically calibrated against several absolute frequency standards at different operating wavelengths, including calibrations against a Cs clock with the aid of an optical frequency comb. This wavemeter also measures the correct frequency that includes any chirp-induced frequency offset as verified when using a narrow-band titanium sapphire pulsed laser source with an adjustable cw-pulse frequency offset used in Ref. [14]. The Raman shift, $\omega_{R}=\omega_{P}-\omega_{S}$, is derived from the simultaneous frequency calibrations of both pump $\omega_{P}$ and Stokes $\omega_{S}$ laser frequencies.

The ac-Stark shift for the $Q(1)$ transition is plotted in Fig. 2, panel (a), as a function of the pump $I_{P}$ and Stokes beam $I_{S}$ intensities, and in panel (c) as a function of the total intensity, $I_{P}+I_{S}$, of both pump and Stokes beams. It was established that the extrapolation to the unperturbed zero-intensity frequency by fitting a plane in (a) or a line in (c) yields the same

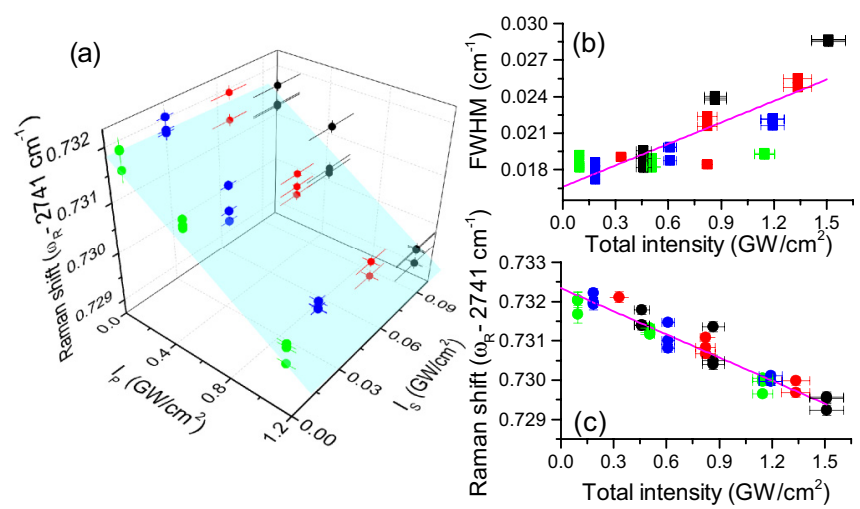

FIG. 2. (a) The ac-Stark extrapolation for the $Q(1)$ line position by fitting a plane (blue-shaded area) spanned by the pump, $I_{P}$, and Stokes, $I_{S}$, laser intensities separately. (b) Full widths at half maximum plotted against the total intensity $I_{P}+I_{S}$. The zero-intensity $\omega_{R}$ value obtained in (a) is consistent with a linear fit in (c) using the total intensity as an independent parameter.

value within $2 \mathrm{MHz}$. This linear dependence is expected since the polarizabilities at $\lambda_{P}$ and $\lambda_{S}$ for both the $v=0$ and $v=1$ levels of molecular hydrogen are very similar [38]. In this manner, the ac-Stark dependence on the total intensity for all other $Q(J=0,2-5)$ transitions was treated by linear extrapolation and found to be accurate to $6 \mathrm{MHz}$. The full widths at half maximum are plotted in Fig. 2(b), which extrapolates to the Doppler width at zero intensity. Collisional shifts on the DT transitions are conservatively estimated to be $\sim 1 \mathrm{MHz}$ (for DT: $\mathrm{D}_{2}: \mathrm{T}_{2}$ partial pressures of 4:8:0.5 mbar), based on investigations of the stable molecular hydrogen species [39-41]. Since we cannot vary the pressure of DT, we have verified the estimates by pressure-dependent measurements of $\mathrm{D}_{2}$ in an identical gas cell. The hyperfine structure of DT is expected to be similar to that of HD with the hyperfine splittings spanning within $\sim 1 \mathrm{MHz}$, and is not observed in our Doppler-limited linewidths. Possible shifts in the hyperfine center of gravity of the transitions are expected to be well below a $\mathrm{MHz}$ and are neglected. Table I shows the uncertainty contributions, where a final uncertainty of $12 \mathrm{MHz}$ or $4 \times 10^{-4} \mathrm{~cm}^{-1}$ is estimated for the $Q(J=0,2-5)$ lines. The $Q(1)$ transition has a slightly smaller uncertainty due to more measurements collected for the assessment of systematic shifts. The reproducibility of

TABLE I. Systematic and statistical contributions to the frequency uncertainties in the DT fundamental vibrational Raman transitions. Values are given in $\mathrm{MHz}$.

\begin{tabular}{lcc}
\hline \hline Contribution & $Q(J \neq 1)$ & $Q(1)$ \\
\hline Pump $\left(\omega_{P}\right)$ calibration & 6 & 6 \\
Stokes $\left(\omega_{S}\right)$ cw calibration & 2 & 1 \\
Stokes cw-pulse chirp correction & 5 & 5 \\
ac-Stark analysis & 6 & 3 \\
Collisional shift & 1 & 1 \\
Statistics & 7 & 5 \\
Combined $(1 \sigma)$ & 12 & 10 \\
\hline \hline
\end{tabular}




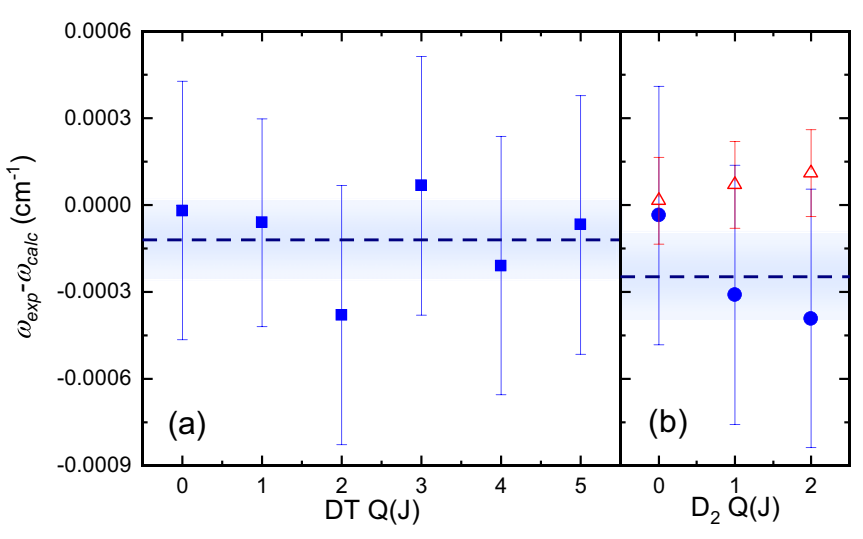

FIG. 3. (a) Comparison of the experimental and calculated DT Raman splittings demonstrating very good agreement. The data points (squares) represent the experiment-calculation differences, $\omega_{\exp }-\omega_{\text {calc }}$, while the error bars represent the combined experimental and theoretical uncertainties. (b) A comparison of $\mathrm{D}_{2} Q(J)$ lines (circles) also measured with the present CARS setup and DT gas cell are shown. In addition, a comparison of $\mathrm{D}_{2}$ molecular beam measurements in Ref. [42] (triangles) is also plotted in (b). The dashed lines indicate the average of experiment-calculation differences (data points) of all measured transitions, while the shaded regions indicate the standard deviation.

measurements performed on multiple days is indicated in the statistics entry in Table I.

Measurements of the $Q(0), Q(1)$, and $Q(2)$ transitions of the fundamental band of $\mathrm{D}_{2}$ with 8-mbar partial pressure inside the same DT cell were also performed. High-accuracy $\mathrm{D}_{2}$ measurements using molecular beams have been performed with a completely different spectroscopic approach [16,42], allowing for the in situ assessment of any other systematic effects. $\mathrm{D}_{2}$ comparisons with Ref. [42] yield an average deviation, shown in Fig. 3, that is consistent with and validates the independently estimated uncertainty of the present CARS study. These experimental results are compared with the theoretical results presented in the following discussion.

\section{THEORY}

In order to calculate molecular rovibrational levels and transition energies accurately, we use a variant of nonrelativistic quantum electrodynamics (NRQED) - an effective theory approximating QED at low-energy scales [12]. It assumes an expansion of the binding energy in powers of the fine-structure constant $\alpha$,

$$
E(\alpha)=\alpha^{2} E^{(2)}+\alpha^{4} E^{(4)}+\alpha^{5} E^{(5)}+\alpha^{6} E^{(6)}+\cdots,
$$

where $E^{(i)}$ is a contribution of order $\alpha^{i} m$ (with the electron mass $m$ ) and may include powers of $\ln \alpha$. Each $E^{(i)}$ can be expressed as an expectation value of some effective Hamiltonian with the nonrelativistic wave function. The $E^{(i)}$ terms can be calculated directly (see, e.g., [10,43-45]) or expanded further in the $m / \mu_{n}$ mass ratio [with $\mu_{n}=M_{A} M_{B} /\left(M_{A}+M_{B}\right)$ the nuclear reduced mass] in the spirit of the nonadiabatic perturbation theory (NAPT) $[9,46]$. This yields the wellknown components of the nonrelativistic energy-the BornOppenheimer (BO) energy $E^{(2,0)}$, the adiabatic correction
$E^{(2,1)}$, and the nonadiabatic correction $E^{(2,2)}$ for a given rovibronic state. Similarly, NAPT enables the relativistic $E^{(4)}$ and QED $E^{(5)}$ corrections to be evaluated as a sum of the leading (infinite nuclear mass) and the recoil (finite mass) components, although finite mass QED corrections have not yet been incorporated.

The Schrödinger equation for a hydrogen molecule isotopologue, written in a center-of-mass frame, with the origin in the geometric center of the nuclei, is

$$
\left(H+H_{n}-E^{(2)}\right)\left|\Psi\left(\vec{r}_{1}, \vec{r}_{2}, \vec{R}\right)\right\rangle=0,
$$

where

$$
\begin{gathered}
H=-\frac{1}{2}\left(\vec{\nabla}_{1}^{2}+\vec{\nabla}_{2}^{2}\right)+V, \\
V=-\frac{1}{r_{1 A}}-\frac{1}{r_{1 B}}-\frac{1}{r_{2 A}}-\frac{1}{r_{2 B}}+\frac{1}{r_{12}}+\frac{1}{R}, \\
H_{n}=-\frac{1}{2 \mu_{n}}\left(\vec{\nabla}_{R}^{2}+\vec{\nabla}_{\mathrm{el}}^{2}\right)+\left(\frac{1}{M_{A}}-\frac{1}{M_{B}}\right) \vec{\nabla}_{R} \vec{\nabla}_{\mathrm{el}},
\end{gathered}
$$

and where the 1,2 indices denote electrons, $A, B$ denote nuclei, $\vec{R}=\vec{R}_{A}-\vec{R}_{B}$, and $\vec{\nabla}_{\mathrm{el}}=\left(\vec{\nabla}_{1}+\vec{\nabla}_{2}\right) / 2$. The last term in $H_{n}$ is present in heteronuclear isotopologues and is a source of the gerade/ungerade mixing effects, of relevance to the DT species investigated here. Within NAPT, the wave function is represented as

$$
\Psi\left(\vec{r}_{1}, \vec{r}_{2}, \vec{R}\right)=\psi\left(\vec{r}_{1}, \vec{r}_{2}\right) Y(\vec{n}) \chi(R) / R+\delta \Psi\left(\vec{r}_{1}, \vec{r}_{2}, \vec{R}\right),
$$

where one assumes $\langle\delta \Psi \mid \psi\rangle_{\mathrm{el}}=0$ (integration over electronic coordinates only), $Y(\vec{n})$ is a spherical harmonic, and $\vec{n}=$ $\vec{R} / R$. Here $\psi\left(\vec{r}_{1}, \vec{r}_{2}\right)$ is an eigenfunction of the electronic Schrödinger equation,

$$
H|\psi\rangle=\mathcal{E}^{(2,0)}(R)|\psi\rangle,
$$

with the eigenvalue dependent on the internuclear distance $R$. In the NAPT leading order, the function $\chi$ satisfies the following nuclear equation,

$$
\begin{gathered}
H_{N} \chi(R)=E^{(2,0)} \chi(R), \\
H_{N}=-\frac{1}{2 \mu_{n}} \frac{d^{2}}{d R^{2}}+\mathcal{E}^{(2,0)}(R)+\frac{J(J+1)}{2 \mu_{n} R^{2}},
\end{gathered}
$$

where $J$ is the rotational quantum number. $\mathcal{E}^{(2,0)}(R)$ from the electronic Schrödinger equation (7) serves as a potential for the movement of the nuclei, present in the nuclear equation (8). We solve Eq. (8) with a discrete variable representation method $[47,48]$ for $\chi$, which is then used in a perturbative manner to calculate the rovibrational energy contributions $\left\langle\chi\left|\mathcal{E}^{(i, k)}(R)\right| \chi\right\rangle$, where $\mathcal{E}^{(i, k)}(R)$ denotes a correction of the order $\alpha^{i} m\left(m / \mu_{n}\right)^{k}$ to the electronic potential. Regarding $E^{(2)}$, its $\mathrm{BO}$ approximation $E^{(2,0)}$ is surely not accurate enough for our purposes. At the same time, direct calculation of $E^{(2,1)}$ and $E^{(2,2)}$ is not very convenient. This is why the nuclear Schrödinger equation is solved again instead-with the NAPT-corrected Hamiltonian, including the nonadiabatic effects up to the $\left(m / \mu_{n}\right)^{2}$ level

$$
\tilde{H}_{N}=\left[-\frac{d}{d R} \frac{1}{2 \mu_{\|}(R)} \frac{d}{d R}+\frac{J(J+1)}{2 \mu_{\perp}(R) R^{2}}+\frac{\mathcal{W}_{\|}^{\prime}(R)}{R}+\mathcal{Y}(R)\right] .
$$


TABLE II. Calculated contributions to the $Q(1)$ transition energy (in units of $\mathrm{cm}^{-1}$ ) in the fundamental band of tritium-bearing molecular hydrogen. $E_{\mathrm{FS}}$ is the finite nuclear size correction with $r_{\mathrm{p}}=0.8414(19) \mathrm{fm}$ [52], $r_{\mathrm{d}}=2.12799(74) \mathrm{fm}$ [52], and $r_{\mathrm{t}}=1.7591(363)$ fm [53], for the proton, deuteron, and triton sizes, respectively. The fine-structure constant $\alpha$, Rydberg constant $R_{\infty}$, and Bohr radius $a_{0}$ are taken from [52], as are the respective proton, deuteron, and triton electron mass ratios: $m_{\mathrm{p}} / m=1836.15267343(11), m_{\mathrm{d}} / m=3670.48296788(13)$, $m_{\mathrm{t}} / m=5496.92153573(27)$.

\begin{tabular}{lccc}
\hline \hline Contribution & $\mathrm{T}_{2}$ & $\mathrm{DT}$ & $\mathrm{HT}$ \\
\hline$E^{(2)}$ & $2463.346322(61)$ & $2741.72999(11)$ & $3431.57340(44)$ \\
$E^{(4)}$ & $0.0148375(1)$ & $0.0163396(1)$ & $0.0198906(1)$ \\
$E^{(4,0)}$ & 0.01480683 & 0.01629689 & 0.01980422 \\
$E^{(4,1)}$ & 0.00003062 & 0.00004272 & 0.00008636 \\
$E^{(5)}$ & $-0.0126866(79)$ & $-0.0141052(96)$ & $-0.0176069(156)$ \\
$E^{(6)}$ & $-0.0001135(3)$ & $-0.0001262(4)$ & $-0.0001578(5)$ \\
$E^{(7)}$ & $0.0000061(15)$ & $0.0000068(17)$ & $0.0000085(21)$ \\
$E_{\mathrm{FS}}$ & $-0.0000082(3)$ & $-0.0000113(2)$ & $-0.0000070(2)$ \\
Total & $2463.348358(62)$ & $2741.73209(11)$ & $3431.57553(44)$ \\
\hline \hline
\end{tabular}

All the functions $\mu_{\|}(R), \mu_{\perp}(R), \mathcal{W}_{\|}^{\prime}(R)$, and $\mathcal{Y}(R)$ in the above are defined and provided as analytic fits in Refs. $[9,46,48]$. Note that $\mathcal{Y}(R)$ incorporates both the adiabatic and nonadiabatic effects, and the $\mathrm{g} / \mathrm{u}$-mixing term from Eq. (5) in particular.

The $E^{(4)}$ and $E^{(6)}$ corrections are calculated as

$$
\begin{gathered}
E^{(4)}=E^{(4,0)}+E^{(4,1)}, \\
E^{(6)}=\left\langle\chi\left|\mathcal{E}^{(6,0)}(R)\right| \chi\right\rangle \\
+\left\langle\chi\left|\mathcal{E}^{(4,0)}(R) \frac{1}{\left(E^{(2,0)}-H_{N}\right)^{\prime}} \mathcal{E}^{(4,0)}(R)\right| \chi\right\rangle,
\end{gathered}
$$

where

$$
\begin{gathered}
E^{(4,0)}=\left\langle\chi\left|\mathcal{E}^{(4,0)}(R)\right| \chi\right\rangle \\
E^{(4,1)}=\left\langle\chi\left|\mathcal{E}^{(4,1)}(R)\right| \chi\right\rangle \\
+2\left\langle\chi\left|\mathcal{E}^{(4,0)}(R) \frac{1}{\left(E^{(2,0)}-H_{N}\right)^{\prime}} \mathcal{E}^{(2,1)}(R)\right| \chi\right\rangle .
\end{gathered}
$$

The rest: $E^{(5)}, E^{(7)}$, and $E_{\mathrm{FS}}$ (the correction due to the finite nuclear sizes) are calculated just as expectation values with $\chi(R)$. All needed potentials are taken from the following: $\mathcal{E}^{(2,0)}(R)$, Refs. [8,48]; $\mathcal{E}^{(2,1)}(R)$, Ref. [49]; the nonadiabatic potentials in Eq. (10), Refs. [9,48]; $\mathcal{E}^{(4,0)}(R)$, Ref. [11]; $\mathcal{E}^{(4,1)}(R)$, Ref. [13]; $\mathcal{E}^{(6,0)}(R)$, Ref. [12]. The potentials $\mathcal{E}^{(7,0)}(R)$ and $\mathcal{E}_{\mathrm{FS}}(R)$ make use of the electron-nucleus Dirac $\delta$ values obtained in Ref. [11]. Their calculation method follows the approach of Refs. $[13,45]$, respectively (based, in turn, on Ref. [50]). The leading QED contribution $\mathcal{E}^{(5,0)}(R)$ combines results from Refs. [11,12,51]. More details concerning particular potentials are available in Ref. [48]. The theoretical uncertainty of an $E^{(i)}$ component includes the missing next term $E^{\left(i, k_{\max }+1\right)} \approx E^{\left(i, k_{\max }\right)} \mathrm{m} / \mu_{n}$, where $k_{\max }$ is the highest included term in the $m / \mu_{n}$ expansion. For $E^{(2)}$, $E^{\left(i, k_{\max }\right)}$ is $E^{(2,2)} \approx E^{(2)}-\left\langle\chi\left|\mathcal{E}^{(2,1)}(R)\right| \chi\right\rangle-E^{(2,0)}$; for $E^{(4)}$ it is $E^{(4,1)}$; and $E^{\left(i, k_{\max }\right)}=E^{(i, 0)}=E^{(i)}$ for $i=5$ and 6 , as well as for $E_{\mathrm{FS}}$. The uncertainties also include an estimate of the numerical error of the respective potential used, and the $E_{\mathrm{FS}}$ error comprises a contribution from uncertainties of the nuclear radii. For $E^{(7)}$, for which only an approximate formula is known, a relative $25 \%$ error is used instead [45].
Total theoretical uncertainties of all the transitions reported in this paper are dominated by the missing $E^{(2,3)}$ in the nonrelativistic contribution. The calculated contributions to the $Q(1)$ transition energy in the fundamental vibrational splitting $(v=0 \rightarrow 1)$ for all tritiated species DT, HT, and $\mathrm{T}_{2}$ are listed in Table II. The theoretical results obtained here are in agreement with Ref. [30], with the present accuracy of $0.00011 \mathrm{~cm}^{-1}$ representing more than a 100 -fold improvement. The $0.02 \mathrm{~cm}^{-1}$ uncertainty in Ref. [30] is dominated by the uncertainty in the nonadiabatic nonrelativistic correction $\left(E^{(2,2)}\right)$, and only includes leading-order relativistic $\left(E^{(4,0)}\right)$ and QED corrections using a Bethe logarithm that is $20 \%$ off from the modern value, or a deviation of $>0.001 \mathrm{~cm}^{-1}$ in $E^{(5)}$

\section{RESULTS, DISCUSSIONS AND CONCLUSION}

The experimental and theoretical values for $Q(J=0-5)$ transition energies in DT are listed in Table III. The present measurements with $0.0004 \mathrm{~cm}^{-1}$ accuracy are consistent with, but are two orders of magnitude more precise than, the previous investigation in Ref. [28]. The comparison of the present experimental and theoretical values demonstrates excellent agreement as listed in Table III and shown graphically in Fig. 3, with the differences (indicated by data points) falling well within $1 \sigma$ of the combined measurement and calculation uncertainty (represented by the error bars).

TABLE III. Fundamental vibrational $(v=0 \rightarrow 1)$ splittings of the $Q(J)$ transitions in DT. The measured values appear in the second column while the theoretical values are listed in the third column, with uncertainties indicated within parentheses. The last column is the difference $\left(\omega_{\exp }-\omega_{\text {calc }}\right)$ with the combined experimentcalculation uncertainty indicated. All values in units of $\mathrm{cm}^{-1}$.

\begin{tabular}{cccc}
\hline \hline Line & Experiment & Calculation & Difference \\
\hline$Q(0)$ & $2743.34171(40)$ & $2743.34174(11)$ & $-0.00003(41)$ \\
$Q(1)$ & $2741.73204(33)$ & $2741.73209(11)$ & $-0.00005(35)$ \\
$Q(2)$ & $2738.51659(40)$ & $2738.51697(11)$ & $-0.00038(41)$ \\
$Q(3)$ & $2733.70470(40)$ & $2733.70466(11)$ & $+0.00004(41)$ \\
$Q(4)$ & $2727.30734(40)$ & $2727.30755(11)$ & $-0.00021(41)$ \\
$Q(5)$ & $2719.34193(40)$ & $2719.34202(11)$ & $-0.00009(41)$ \\
\hline \hline
\end{tabular}


In summary, we have determined $Q(J=0-5)$ transition energies of the fundamental band of DT with a 250 -fold improvement over all other previous measurements. Highly accurate calculations are also presented with similar improvements of uncertainty. Studies using the heavier tritiated species are useful in disentangling various mass-dependent effects that have been frequently overlooked in the literature [54]. In view of a present $2.7 \sigma$ discrepancy in experiment and calculations in the dissociation energy of HD [44] while there is perfect agreement for $\mathrm{H}_{2}$ and $\mathrm{D}_{2}$, investigations on heteronuclear species such as DT may be helpful in the resolution of the HD discrepancy.

Since the present experimental and theoretical values are in very good agreement, these can be used to constrain hypothetical long-range fifth forces between hadrons [55]. In fifthforce investigations on diatomic molecules, the hypothetical interaction is parametrized as a Yukawa potential,

$$
V_{5}\left(R ; \alpha_{5}, \lambda_{5}\right)=N_{1} N_{2} \frac{\alpha_{5} \exp \left(-R / \lambda_{5}\right)}{R} \hbar c,
$$

where $\alpha_{5}$ and $\lambda_{5}$ are the interaction strength and length parameters, respectively, while $R$ is the distance between the two nuclei of nuclear numbers $N_{1}$ and $N_{2}$. The most stringent constraint for these hypothetical fifth forces in the range $\lambda_{5} \sim 1 \AA$ is derived from $\mathrm{HD}^{+}[56]$ yielding $\alpha_{5}<8 \times 10^{-10} \alpha$, where $\alpha$ is the electromagnetic coupling strength. This tight bound is largely due to the sub-MHz uncertainties obtained in the $\mathrm{HD}^{+}$measurements. Applying the method in Ref. [55] on the DT $Q(1)$ transition, the strength of a fifth force for an interaction range of $\sim 1 \AA$ is constrained at $\alpha_{5}<2 \times 10^{-8} \alpha$, which is more than an order of magnitude weaker than the $\mathrm{HD}^{+}$ derived bound. With the sixfold enhanced sensitivity of DT with respect to the lightest species $\mathrm{H}_{2}$ as seen from Eq. (15), the present DT results yield a limit that is slightly more stringent than that of $\mathrm{H}_{2}$. This is despite the $\mathrm{H}_{2}(v=0 \rightarrow 1)$ $Q$ lines [16,42] having 2.7 times better accuracy than the present DT study. With the inherent sensitivity of the heavier tritiated species (with $N_{t}=3$ ) this limit can be further tightened when an accuracy in the $\mathrm{kHz}$ level is reached, for example by using techniques recently applied to HD [25].

\section{ACKNOWLEDGMENTS}

We are grateful to Tobias Falke, David Hillesheimer, Stefan Welte, and Jürgen Wendel of TLK for the preparation and handling of the tritium cell. W.U. thanks the European Research Council for ERC-Advanced Grant No. 670168. The calculations were supported by the National Science Center (Poland), Grants No. 2017/27/B/ST2/02459 (K.P.) and No. 2016/23/B/ST4/01821 (M.P.), and by PL-Grid Infrastructure. The research leading to these results has received funding from Laserlab-Europe (Grant Agreement No. 654148, the European Union's Horizon 2020 research and innovation program).
[1] D. Hanneke, S. Fogwell, and G. Gabrielse, Phys. Rev. Lett. 100, 120801 (2008).

[2] R. Bouchendira, P. Cladé, S. Guellati-Khélifa, F. Nez, and F. Biraben, Phys. Rev. Lett. 106, 080801 (2011).

[3] R. H. Parker, C. Yu, W. Zhong, B. Estey, and H. Müller, Science 360, 191 (2018).

[4] C. G. Parthey, A. Matveev, J. Alnis, B. Bernhardt, A. Beyer, R. Holzwarth, A. Maistrou, R. Pohl, K. Predehl, T. Udem, T. Wilken, N. Kolachevsky, M. Abgrall, D. Rovera, C. Salomon, P. Laurent, and T. W. Hänsch, Phys. Rev. Lett. 107, 203001 (2011).

[5] R. Pohl, A. Antognini, F. Nez, F. D. Amaro, F. Biraben, J. M. R. Cardoso, D. S. Covita, A. Dax, S. Dhawan, L. M. P. Fernandes, A. Giesen, T. Graf, T. W. Hänsch, P. Indelicato, L. Julien, C. Y. Kao, P. Knowles, E.-O. L. Bigot, Y.-W. Liu, J. A. M. Lopes, L. Ludhova, C. M. B. Monteiro, F. Mulhauser, T. Nebel, P. Rabinowitz, J. M. F. dos Santos, L. A. Schaller, K. Schuhmann, C. Schwob, D. Taqqu, J. F. C. A. Veloso, and F. Kottmann, Nature (London) 466, 213 (2010).

[6] J. Shapiro and G. Breit, Phys. Rev. 113, 179 (1959).

[7] J. H. Black and A. Dalgarno, Astrophys. J. 203, 132 (1976).

[8] K. Pachucki, Phys. Rev. A 82, 032509 (2010).

[9] K. Pachucki and J. Komasa, J. Chem. Phys. 143, 034111 (2015).

[10] K. Pachucki and J. Komasa, Phys. Chem. Chem. Phys. 20, 247 (2018).

[11] M. Puchalski, J. Komasa, and K. Pachucki, Phys. Rev. A 95, 052506 (2017).

[12] M. Puchalski, J. Komasa, P. Czachorowski, and K. Pachucki, Phys. Rev. Lett. 117, 263002 (2016).
[13] P. Czachorowski, M. Puchalski, J. Komasa, and K. Pachucki, Phys. Rev. A 98, 052506 (2018).

[14] C.-F. Cheng, J. Hussels, M. Niu, H. L. Bethlem, K. S. E. Eikema, E. J. Salumbides, W. Ubachs, M. Beyer, N. Hölsch, J. A. Agner, F. Merkt, L.-G. Tao, S.-M. Hu, and C. Jungen, Phys. Rev. Lett. 121, 013001 (2018).

[15] N. Hölsch, M. Beyer, E. J. Salumbides, K. S. E. Eikema, W. Ubachs, C. Jungen, and F. Merkt, Phys. Rev. Lett. 122, 103002 (2019).

[16] G. D. Dickenson, M. L. Niu, E. J. Salumbides, J. Komasa, K. S. E. Eikema, K. Pachucki, and W. Ubachs, Phys. Rev. Lett. 110, 193601 (2013).

[17] A. Campargue, S. Kassi, K. Pachucki, and J. Komasa, Phys. Chem. Chem. Phys. 14, 802 (2012).

[18] S. Kassi and A. Campargue, J. Mol. Spectrosc. 300, 55 (2014).

[19] C.-F. Cheng, Y. R. Sun, H. Pan, J. Wang, A.-W. Liu, A. Campargue, and S.-M. Hu, Phys. Rev. A 85, 024501 (2012).

[20] J. Liu, D. Sprecher, C. Jungen, W. Ubachs, and F. Merkt, J. Chem. Phys. 132, 154301 (2010).

[21] P. Maddaloni, P. Malara, E. D. Tommasi, M. D. Rosa, I. Ricciardi, G. Gagliardi, F. Tamassia, G. D. Lonardo, and P. D. Natale, J. Chem. Phys. 133, 154317 (2010).

[22] S. Kassi, A. Campargue, K. Pachucki, and J. Komasa, J. Chem. Phys. 136, 184309 (2012).

[23] S. Kassi and A. Campargue, J. Mol. Spectrosc. 267, 36 (2011).

[24] L.-G. Tao, A.-W. Liu, K. Pachucki, J. Komasa, Y. R. Sun, J. Wang, and S.-M. Hu, Phys. Rev. Lett. 120, 153001 (2018).

[25] F. M. J. Cozijn, P. Dupré, E. J. Salumbides, K. S. E. Eikema, and W. Ubachs, Phys. Rev. Lett. 120, 153002 (2018). 
[26] E. Fasci, A. Castrillo, H. Dinesan, S. Gravina, L. Moretti, and L. Gianfrani, Phys. Rev. A 98, 022516 (2018).

[27] H. G. M. Edwards, D. A. Long, and H. R. Mansour, J. Chem. Soc., Faraday Trans. 2 74, 1203 (1978).

[28] D. Veirs and G. Rosenblatt, J. Mol. Spectrosc. 121, 401 (1987).

[29] M.-C. Chuang and R. N. Zare, J. Mol. Spectrosc. 121, 380 (1987).

[30] C. Schwartz and R. J. Le Roy, J. Mol. Spectrosc. 121, 420 (1987).

[31] T. M. Trivikram, M. Schlösser, W. Ubachs, and E. J. Salumbides, Phys. Rev. Lett. 120, 163002 (2018).

[32] K. S. E. Eikema, W. Ubachs, W. Vassen, and W. Hogervorst, Phys. Rev. A 55, 1866 (1997).

[33] M. Schlösser, X. Zhao, M. T. Trivikram, W. Ubachs, and E. J. Salumbides, J. Phys. B 50, 214004 (2017).

[34] S. Xu, R. van Dierendonck, W. Hogervorst, and W. Ubachs, J. Mol. Spectrosc. 201, 256 (2000).

[35] M. S. Fee, K. Danzmann, and S. Chu, Phys. Rev. A 45, 4911 (1992).

[36] S. Gangopadhyay, N. Melikechi, and E. E. Eyler, J. Opt. Soc. Am. B 11, 231 (1994).

[37] S. Hannemann, E. J. Salumbides, S. Witte, R. T. Zinkstok, E. J. van Duijn, K. S. E. Eikema, and W. Ubachs, Phys. Rev. A 74, 062514 (2006).

[38] M. J. Dyer and W. K. Bischel, Phys. Rev. A 44, 3138 (1991).

[39] L. A. Rahn and G. J. Rosasco, Phys. Rev. A 41, 3698 (1990).

[40] G. J. Rosasco, A. D. May, W. S. Hurst, L. B. Petway, and K. C. Smyth, J. Chem. Phys. 90, 2115 (1989).

[41] G. J. Rosasco, W. J. Bowers, W. S. Hurst, J. P. Looney, K. C. Smyth, and A. D. May, J. Chem. Phys. 94, 7625 (1991).
[42] M. L. Niu, E. J. Salumbides, G. D. Dickenson, K. S. E. Eikema, and W. Ubachs, J. Mol. Spectrosc. 300, 44 (2014).

[43] L. M. Wang and Z.-C. Yan, Phys. Rev. A 97, 060501(R) (2018).

[44] M. Puchalski, A. Spyszkiewicz, J. Komasa, and K. Pachucki, Phys. Rev. Lett. 121, 073001 (2018).

[45] M. Puchalski, J. Komasa, P. Czachorowski, and K. Pachucki, Phys. Rev. Lett. 122, 103003 (2019).

[46] K. Pachucki and J. Komasa, Phys. Chem. Chem. Phys. 12, 9188 (2010).

[47] D. T. Colbert and W. H. Miller, J. Chem. Phys. 96, 1982 (1992).

[48] J. Komasa, M. Puchalski, P. Czachorowski, G. Łach, and K. Pachucki, Phys. Rev. A 100, 032519 (2019).

[49] K. Pachucki and J. Komasa, J. Chem. Phys. 141, 224103 (2014).

[50] M. Eides, H. Grotch, and V. Shelyuto, Phys. Rep. 342, 63 (2001).

[51] K. Piszczatowski, G. Łach, M. Przybytek, J. Komasa, K. Pachucki, and B. Jeziorski, J. Chem. Theory Comput. 5, 3039 (2009).

[52] CODATA 2018 recommended values, https://physics.nist.gov/ cuu/Constants.

[53] I. Angeli and K. Marinova, At. Data Nucl. Data Tables 99, 69 (2013).

[54] M. Puchalski, J. Komasa, and K. Pachucki, Phys. Rev. Lett. 120, 083001 (2018).

[55] E. J. Salumbides, J. C. J. Koelemeij, J. Komasa, K. Pachucki, K. S. E. Eikema, and W. Ubachs, Phys. Rev. D 87, 112008 (2013).

[56] J. Biesheuvel, J.-Ph. Karr, L. Hilico, K. S. E. Eikema, W. Ubachs, and J. C. J. Koelemeij, Nat. Commun. 7, 10385 (2016). 